\title{
Glanzmann thrombasthenia: genetic basis and clinical correlates
}

Haematologica 2020

Volume 105(4):888-894

\author{
Juliana Perez Botero, ${ }^{1}$ Kristy Lee, ${ }^{2}$ Brian R Branchford, ${ }^{3}$ Paul F Bray, ${ }^{4}$ Kathleen \\ Freson, ${ }^{5}$ Michele P. Lambert, ${ }^{6}$ Minjie Luo, ${ }^{7}$ Shruthi Mohan, ${ }^{2}$ Justyne E. Ross, ${ }^{2}$ \\ Wolfgang Bergmeier ${ }^{8}$ and Jorge Di Paola ${ }^{9}$ on behalf of the ClinGen Platelet \\ Disorder Variant Curation Expert Panel
}

${ }^{1}$ Versiti and Division of Hematology/Oncology, Medical College of Wisconsin, Milwaukee, WI, USA; ${ }^{2}$ Department of Genetics, University of North Carolina at Chapel Hill, NC, USA; ${ }^{3}$ University of Colorado School of Medicine, Department of Pediatrics, Division of Hematology/Oncology/Bone Marrow Transplant, Aurora, CO, USA; ${ }^{4}$ Molecular Medicine Program, Division of Hematology and Hematologic Malignancies, Department of Medicine, University of Utah, Salt Lake City, UT, USA; ${ }^{5}$ Center for Molecular and Vascular Biology, University of Leuven, Leuven, Belgium; ${ }^{\top}$ The Children's Hospital of Philadelphia and Department of Pediatrics, Perelman School of Medicine, Philadelphia, PA, USA; ${ }^{7}$ Department of Pathology and Laboratory Medicine, The Children's Hospital of Philadelphia, Perelman School of Medicine, Philadelphia, PA, USA; ${ }^{8}$ Department of Biochemistry and Biophysics and UNC Blood Research Center, University of North Carolina at Chapel Hill, NC, USA and ${ }^{9}$ Division of Pediatric Hematology Oncology, Department of Pediatrics, Washington University School of Medicine in St. Louis, MO, USA

\section{Correspondence: \\ JORGE DI PAOLA \\ dipaolaj@wustl.edu \\ Received: December 18, 2019. \\ Accepted: February 7, 2020. \\ Pre-published: March 5, 2020.}

doi:10.3324/haematol.2018.214239

Check the online version for the most updated information on this article, online supplements, and information on authorship \& disclosures: www.haematologica.org/content/105/4/888

\section{(C)2020 Ferrata Storti Foundation}

Material published in Haematologica is covered by copyright. All rights are reserved to the Ferrata Storti Foundation. Use of published material is allowed under the following terms and conditions:

https://creativecommons.org/licenses/by-nc/4.0/legalcode. Copies of published material are allowed for personal or internal use. Sharing published material for non-commercial purposes is subject to the following conditions: https://creativecommons. org//licenses/by-nc/4.0/legalcode, sect. 3. Reproducing and sharing published material for commercial purposes is not allowed without permission in writing from the publisher.

\section{ABSTRACT}

lanzmann thrombasthenia (GT) is an autosomal recessive disorder of platelet aggregation caused by quantitative or qualitative defects in integrins $\alpha \mathrm{IIb}$ and $\beta 3$. These integrins are encoded by the ITGA2B and ITGB3 genes and form platelet glycoprotein (GP)IIb/IIIa, which acts as the principal platelet receptor for fibrinogen. Although there is variability in the clinical phenotype, most patients present with severe mucocutaneous bleeding at an early age. A classic pattern of abnormal platelet aggregation, platelet glycoprotein expression and molecular studies confirm the diagnosis. Management of bleeding is based on a combination of hemostatic agents including recombinant activated factor VII with or without platelet transfusions and antifibrinolytic agents. Refractory bleeding and platelet alloimmunization are common complications. In addition, pregnant patients pose unique management challenges. This review highlights clinical and molecular aspects in the approach to patients with GT, with particular emphasis on the significance of multidisciplinary care.

\section{Introduction}

The Swiss pediatrician Eduard Glanzmann was the first to describe in 1918 a type of purpura that presented with normal platelet count and size in patients with absent/decreased clot retraction and prolonged bleeding time. ${ }^{1}$ In 1962, Caen and Cousin described the lack of platelet aggregation with multiple agonists, ${ }^{2}$ and a few years later correlated the platelet aggregation result with decreased platelet fibrinogen and a slight reduction in clot retraction in individuals with Glanzmann thrombasthenia (GT). ${ }^{3}$ The lack of one of the three major platelet surface glycoproteins in GT was reported by Nurden and Caen in 1974; ${ }^{4}$ several other scientists subsequently identified this as the glycoprotein (GP) IIb/IIIa complex. Additional studies with more advanced glycoprotein imaging techniques provided evidence for the definition of two disease groups: type I with absent IIb/IIIa expression $(<5 \%$ of normal) and type II with reduced expression $(5-20 \%$ of normal GP IIb/IIIa). ${ }^{5} \mathrm{~A}$ third type (type III) has normal levels of integrin but the protein is nonfunctional.

Glanzmann thrombasthenia is considered a rare disease, defined in the United States as those affecting less than 200,000 individuals. ${ }^{6}$ The exact incidence has been difficult to calculate, but is estimated at one in 1,000,000. With an autosomal recessive inheritance, males and females are affected equally. There is a world- 
wide distribution; however, a large proportion of the cases have been described in selected populations such as the French Romani, ${ }^{7}$ South Indian Hindus, Iraqi Jews, and Jordanian nomadic tribes, ${ }^{8}$ in all of which consanguinity is common. Type I is the most common subtype and accounts for around $78 \%$ of patients with GT type II and type III (functional variant in receptor) constituting around $14 \%$ and $8 \%$ of cases, respectively. ${ }^{9}$

GPIIb/IIIa (integrin $\alpha \operatorname{IIb} \beta 3$ ) is a heterodimeric receptor present in large quantities in the plasma membrane of platelets. Activation of this integrin and binding of soluble ligands [primarily fibrinogen, but also von Willebrand factor (vWF) and fibronectin] are essential for platelet aggregation. In the resting state, the integrin has low affinity for ligands. During platelet activation, driven by exposure to soluble agonists or the subendothelial matrix, "inside out" cellular signaling generates a conformational change in GPIIb/IIIa that allows high affinity binding for fibrinogen, which serves as a "bridge" to other activated platelets, eventually forming the platelet plug. Protein kinase C (PKC), diaglycerol-regulated guanine nucleotide exchange factor I (CalDAG-GEFI or RASGRP2), and phosphoinositide 3-kinase (PI3K) participate in this signaling pathway. Subsequent "outside in" signaling triggers additional granule secretion, cytoskeletal interactions (that allow for platelet spreading), stabilization and clot retraction to consolidate the fibrin clot. Kindlins (including kindlin-3) and talins are key regulators of integrin activation. ${ }^{10,11}$

Glanzmann thrombasthenia is usually caused by decreased or absent expression of $\alpha \mathrm{IIb}$ or $\beta 3$, abnormalities in protein folding, defective post-translational processing or transport of either integrin subunit causing decreased surface expression, or abnormalities affecting protein function. Other defects change integrin function by altering the ligand binding pocket (interface between $\alpha I I b$ and $\beta 3$ ), which modifies the cytoplasmic domain and affects binding of regulators, or locks the integrin in the activated form.

\section{Molecular basis of Glanzmann thrombasthenia}

The ITGA2B and ITGB3 genes are located on chromosomes $17 q 21.31$ and $17 q 21.32$, respectively, and are independently expressed. GT is caused by pathogenic variants in both alleles of either of the two genes; concomitant pathogenic variants in both genes, but affecting only one allele of each, is not known to cause GT. Due to the autosomal recessive inheritance, compound heterozygosity is frequent, except in selected ethnic groups where homozygosity is more likely due to consanguinity. A higher percentage of pathogenic variants occur in ITGA2B, likely because of the larger size of this gene with 30 exons encoding 1,039 amino acids, compared to ITGB3 which is composed of 15 exons with 788 amino acids. The clinical phenotypes associated with either gene are indistinguishable. ${ }^{12}$

Pathogenic nonsense, missense and splice site variants are common and large deletions and duplications, although rare, have also been described. ${ }^{13}$ Pathogenic missense variants impair subunit biosynthesis in megakaryocytes or inhibit the transport of the pro- $\alpha I \mathrm{Il} \beta 3$ complexes from the endoplasmic reticulum (ER) to the Golgi apparatus or the export of the mature complexes to the cell surface. A large proportion of variants affect the $\beta$ - propeller region of $\alpha \mathrm{IIb}$ and the epithelial growth factor domains of $\beta 3 .{ }^{14}$

A different type of variant affecting specific regions of these genes has been more recently described to cause a mild autosomal dominant macrothrombocytopenia ${ }^{15}$ by interfering with proplatelet formation. ${ }^{16}$ These "gain of function" variants cause spontaneous activation of GPIIb/IIIa ( $\alpha I I b \beta 3$ ) by affecting the cytoplasmic domains or the membrane proximal residues in the extracellular domains. The majority of these are in ITGB3 and affect the MIDAS (metal ion dependent adhesion site), ADMIDAS (adjacent to MIDAS) or SyMBS (synergistic metal ion binding site) regions. A small proportion have been reported in ITGA2B and affect the conserved intracellular GFFKR sequence. ${ }^{17}$

\section{Clinical manifestations and diagnosis}

\section{Bleeding phenotype}

With integrins $\alpha I \mathrm{Ib}$ and $\beta 3$ participating in primary hemostasis, the bleeding manifestations are typically purpura, epistaxis (60-80\%), gum bleeding (20-60\%), and menorrhagia (60-90\%). Gastrointestinal bleeding in the form of melena or hematochezia is present in 10-20\%, and $1-2 \%$ develop intracranial hemorrhage. ${ }^{9}$ Mucocutaneous bleeding can be spontaneous or occur after minimal trauma. Epistaxis is the most common cause of severe bleeding, especially in the pediatric population, and risk of severe nosebleeds decreases with age as the septal arterial plexus becomes less friable and children grow out of the habit of nose picking. Menorrhagia is highly prevalent in affected females and there is a higher risk of severe bleeding at the time of menarche due to the prolonged estrogen influence on the proliferative endometrium that occurs during anovulatory cycles. Bleeding complications during pregnancy are uncommon; however, the risk of obstetric hemorrhage at the time of delivery and postpartum is high. Hematuria and spontaneous hemarthrosis have been described in some cases but are not usually part of the bleeding phenotype.

Several bleeding scores have been developed with the goal of standardizing the assessment of bleeding and facilitating the diagnosis of patients with a suspected inherited bleeding disorder. These are useful tools that assist in communication of the bleeding phenotype in the clinical and research setting; however, they have not been widely validated for patients with inherited platelet function disorders. Therefore, specific cutoffs to define a positive bleeding score have not been established for this population. ${ }^{18}$ This is of particular relevance in GT, because while the types of bleeding are consistent among individuals, the degree of bleeding is highly variable. Given the severity of this disorder, historically, most patients have been diagnosed in childhood (before 5 years of age), but there are some patients that reach adulthood without having severe bleeding. ${ }^{9}$ In general, the severity of bleeding (except for menorrhagia and pregnancy-associated bleeding) decreases with age.

\section{Laboratory phenotype \\ Complete blood count}

There should be normal platelet count and size with normal granularity on evaluation of the peripheral blood smear by light microscopy. If bleeding is severe and/or 
chronic, patients can have low hemoglobin, microcytosis and increased red cell distribution width from secondary iron deficiency. Other abnormalities of the complete blood count $(\mathrm{CBC})$, suggest an alternative diagnosis.

\section{Coagulation screening tests}

Routine tests ordered in the workup of a patient with abnormal bleeding, such as prothrombin time (PT), activated thromboplastin time (aPTT) and fibrinogen, are usually normal, unless a patient is being evaluated in the setting of significant acute hemorrhage and has evidence of consumptive coagulopathy.

\section{Platelet function screening tests}

The platelet function analyzer (PFA)-100 provides a measure of platelet function under high shear. It is convenient as it uses low volume whole blood samples and is widely available to clinicians. Very prolonged closure times (>300 seconds) are compatible with GT but not specific, as other disorders, such as severe von Willebrand disease, Bernard Soulier syndrome and afibrinogenemia, can produce the same result. However, a normal PFA-100 has a very high negative predictive value for GT and virtually excludes this diagnosis. ${ }^{19}$

\section{Platelet light transmission aggregometry}

Despite its limited availability and the need for largervolume samples and immediate processing, platelet light transmission aggregometry (LTA) remains the gold standard in the clinical diagnosis of GT. It is based on the decreased turbidity generated by platelet agglutinates or aggregates in platelet rich plasma after exposure to different agonists. Decreased/absent aggregation $(<10 \%)$ with all physiologic agonists, together with a normal agglutination response to ristocetin (mediated by GPIb-IX-V), is the classic pattern observed in patients with GT. ${ }^{20}$ Due to the large variability in platelet aggregation results and the significant effect of pre-analytical variables on this test, confirmation of the findings in a second sample is recommended.

\section{Whole blood impedance aggregometry}

This is available in several centers worldwide. While it can be performed in whole blood samples and using lower volumes, there is not enough evidence to support equivalent sensitivity and reproducibility when compared to LTA. ${ }^{21}$ There is some clinical utility to this test in cases in which access to LTA is difficult; however, patients should preferably be referred for evaluation at a center that has LTA capacity at least once to confirm the diagnosis.

\section{Platelet glycoprotein expression study by flow cytometry}

This assesses quantitative platelet surface glycoprotein deficiencies using fluorescent-conjugated antibodies that are specific towards GP.22 This test can be performed in low sample volumes shipped for analysis; however, it will not identify type III (functional) defects that are caused by qualitative but not quantitative defects in GPIIb/IIIa (Figure 1).

\section{Differential diagnosis \\ Leukocyte adhesion deficiency type III}

Leukocyte adhesion deficiency type III (LAD-III) is an autosomal recessive disorder caused by pathogenic vari- ants in the kindlin 3 gene FERMT ${ }^{23}$ that also presents failure of the "inside-out" integrin activation in platelets, white cells and endothelial cells, ${ }^{11}$ causing bleeding, infections and impaired wound healing. Due to the functional integrin defect affecting platelets, these patients have the same platelet aggregation pattern as those with GT and similar to type III (variant) GT, but have normal platelet glycoprotein expression by flow cytometry. The associated neutrophil dysfunction leading to frequent bacterial infections and impaired wound healing in patients with LAD-III may help clinicians distinguish these patients from those with GT.

\section{RASGRP2 related platelet dysfunction}

RASGRP2 encodes calcium and diacylglycerol-regulated guanine exchange factor I (CalDAG-GEFI), a protein that also participates in "inside out" signaling of integrins. Pathogenic variants in this gene lead to autosomal recessive non-syndromic platelet dysfunction characterized by moderate to severe bleeding and decreased platelet aggregation with ADP and epinephrine, and, in some cases, arachidonic acid, collagen and thrombin. ${ }^{24}$

\section{Bernard Soulier syndrome}

Bernard Soulier syndrome (BSS) is also an autosomal recessive disorder caused by pathogenic variants in $G P 1 B A, G P 1 B B$ and GP9. The clinical presentation in terms of bleeding phenotype is very similar to GT; however, BSS is relatively easy to distinguish due to macrothrombocytopenia, platelet LTA with normal aggregation with all agonists except ristocetin, and protein assessment (e.g. flow cytometry) showing decreased/absent CD42a (GPIX) and CD42b (GPIbalpha).

\section{Acquired Glanzmann thrombasthenia}

Acquired GT is typically caused by antibodies with specificity against GPIIb/IIIa (or nearby epitopes) that block the interaction of the receptor with fibrinogen and von Willebrand factor. It presents with late-onset, severe mucocutaneous bleeding in the setting of normal platelet counts ${ }^{25}$ and is usually secondary to autoimmune, lymphoproliferative or plasma cell disorders. Medications, specifically anti-thrombotics that block GPIIb/IIIa such as abciximab, eptifibatide and tirofiban, have also been implicated. ${ }^{26}$ The absence of lifelong bleeding and presence of concomitant systemic disorder should lead the clinician to suspect this diagnosis.

\footnotetext{
- Normal platelet count, size and granularity on light microscopy

- Platelet light transmission aggregometry with decreased/absent aggregation ( $<10 \%)$ with all agonists except ristocetin

- Platelet glycoprotein expression study by flow cytometry with decreased/absent (<20\%) CD41 (GPIlb) and CD61 (GPIIla)
}

Figure 1. Typical laboratory phenotype in Glanzmann thrombasthenia (GT). 


\section{Molecular confirmation}

Genetic analysis is clinically useful for confirmation of the diagnosis, identification of at-risk carriers, reproductive risk counseling for a given couple/family, and for definitive prenatal or preimplantation genetic diagnosis. Genetic counseling plays an essential role in the genetic testing process, obtaining informed consent regarding the unique considerations, benefits, and limitations of genetic testing, and addressing the complexities of clinical application or molecular findings, unexpected results, variants of uncertain significance, and familial implications for medical risks or biological relationships. Genetic coun-

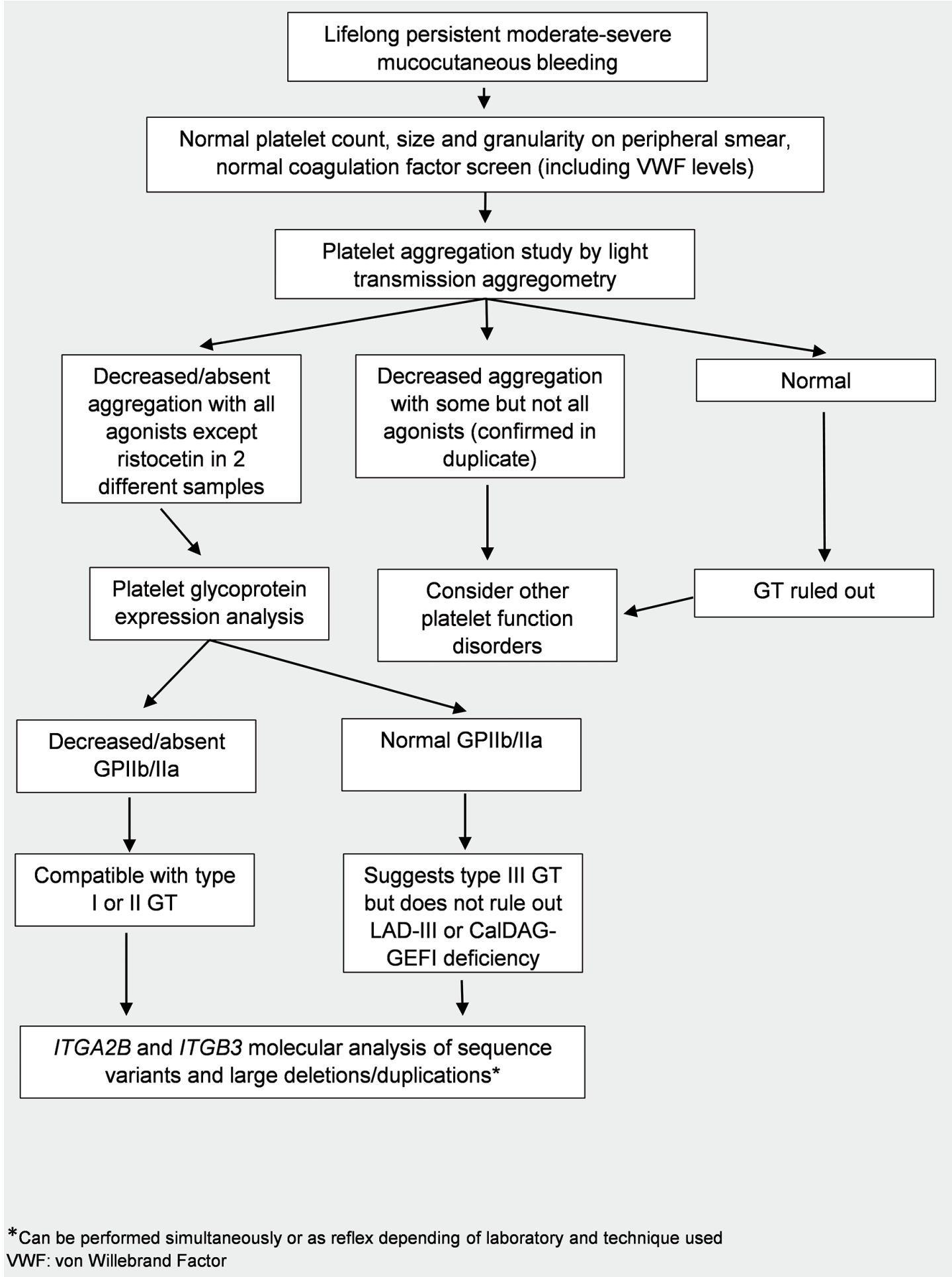

Figure 2. Diagnostic flow chart for the laboratory evaluation of a patient with suspected Glanzmann thrombasthenia (GT). 
selors can support clinicians by providing pre- and posttest genetic counseling to patients and families in the clinical setting, and can also aid clinicians in test selection and ordering, and in navigating the process of obtaining insurance approval for payment. This is particularly relevant in healthcare systems with multiple payers and multiple testing options. As commercial laboratories offer different services, the clinician should be familiar with the testing methodology and any related limitations.

Given the highly specific phenotype of GT, after platelet aggregation studies and flow cytometry have been performed, genetic testing of ITGA2B and ITGB3 only, as opposed to a panel approach that includes multiple other genes, is appropriate (Figure 2). Sequence analysis will detect the vast majority of pathogenic variants; when sequencing fails to identify both pathogenic variants in a patient with GT, specific deletion/duplication analysis should be considered.

Targeted variant analysis, which is expected to save time and money, can be performed in populations with a known pathogenic variant(s) and high rates of consanguinity; however, with this approach, a second GT variant in the same gene may go undetected. ${ }^{27}$ Targeted variant analysis is best adopted when the pathogenic variant in each allele has been identified in an affected individual.

\section{Variant curation and initiatives for standardization}

In recent years, several initiatives have been taken to provide guidance for the analysis and reporting of molecular findings, specifically in terms of variant classification when attributing pathogenicity. In the United States, the American College of Medical Genetics and Genomics (ACMG) and Association for Molecular Pathology (AMP) published guidelines in 2015 for the interpretation of variants, providing a framework for clinical molecular laboratories. ${ }^{14}$ While these guidelines have been extremely useful in the laboratory practice, they are not disease specific, and significant challenges remain when applied to particular disease states. In an effort to address this limitation, and to improve the quality, consistency and access to clinical genomic data, the National Institutes of Health (NIH) funded resource ClinGen is leading initiatives including the formation of Variant Curation Expert Panels (VCEP). Each VCEP consists of a cross-institutional team of experts from complementary specialties, the fields of hematology and genetics, from academia and industry, working collaboratively with the goal of adapting the ACMG criteria for specific genes or disorders. ${ }^{28}$

The Platelet Disorder Variant Curation Expert Panel (VCEP) created in 2018 and supported by the American Society of Hematology, is composed of 28 international scientists and clinicians with expertise in hematology and genetics, and has been working on the adaptation of the ACMG rules for the interpretation of variants in ITGA2B and ITGB3. The goals of this collaboration are to produce high quality variant curation data that are publicly available and create a framework for GT variant curation that allows others to systematically and comprehensively approach genetic data encountered in clinical and research settings.

The VCEP has used estimates of prevalence and population data to define criteria for allele frequency that ultimately serve as standalone, strong or supporting evidence to classify variants as benign, which is a difficult task in rare disorders for which the precise incidence data are not available. Clinical expertise was key in defining the phenotype from a clinical presentation (bleeding phenotype) and clinical laboratory standpoint (platelet aggregation and glycoprotein expression studies), which together are unique to GT. Knowledge of the molecular biology of the disorder allowed for elimination of codes that do not apply for this disease state and points for segregation analysis were modified taking into account the disease inheritance, low frequency and specific clinical phenotype. Special consideration was given to defining the type of assays that provide quality functional evidence in different in vivo and in vitro systems and models. The disease specified rules were tested in a subset of variants which will be uploaded to ClinVar with a 3-star rating. Variants assessed by approved ClinGen VCEP also receive a US Food and Drug Administration (FDA) approval label. A detailed description of the rule specifications for GT will soon be available to the public.

\section{Management}

Patients with GT benefit from being managed at a center with expertise in inherited bleeding disorders, with access to staff who are able to provide recommendations and

Table 1. Measures and medications available for the management of bleeding in Glanzmann thrombasthenia (GT).

\begin{tabular}{|c|c|}
\hline Ireatment & Clinical situation \\
\hline \multicolumn{2}{|c|}{$\begin{array}{l}\text { - Compression } \\
\text { - Gauze or sponge dipped in antifibrinolytic or topical thrombin } \\
\text { - Nasal packing } \\
\text { - Gel foam soaked in antifibrinolytic or topical thrombin }\end{array}$} \\
\hline $\begin{array}{l}\text { Antifibrinolytics } \\
\text { - Tranexamic acid } \\
\text { - Aminocaproic acid }\end{array}$ & $\begin{array}{l}\text { As monotherapy in mild bleeding } \\
\text { As combination therapy in moderate and severe bleeding }\end{array}$ \\
\hline \multirow[t]{2}{*}{ rFVIIa } & Moderate to severe bleeding \\
\hline & $\begin{array}{l}\text { Alone for minor surgical procedures or in combination with antifibrinolytics and/or platelet } \\
\text { transfusions for major procedures and severe or life-threatening bleeding }\end{array}$ \\
\hline Platelet transfusion* & In combination with rFVIIa and/or antifibrinolytics in severe bleeding and major surgical procedures \\
\hline
\end{tabular}

rFVIIa: recombinant activated coagulation factor VII. *Risk of lack of efficacy in the setting of alloimmunization. 
treatment any time of day, and also benefit from being provided directly with medical alerts, and emergency medical contact and treatment information to present when seeking urgent care from clinicians who are not familiar with their case history. Education on avoidance of over-thecounter medications that increase bleeding risk such as non-steroidal anti-inflammatories and aspirin products should be provided. Prescription medications that can affect hemostasis should be carefully monitored. ${ }^{29}$

\section{Hemostatic management}

The treatment of bleeding in patients with GT includes management of acute or chronic bleeding and prevention of hemorrhagic complications around the time of procedures. The choice of treatment depends on the severity of bleeding (Table 1), availability of products, and prior responses to therapy, and is similar in some aspects to the management of patients with other severe bleeding disorders. Note that desmopressin (DDAVP), commonly used in von Willebrand Disease and other milder disorders of platelet function, is of limited clinical utility in the treatment of GT (Table 1).

Recombinant activated factor VII (rFVIIa) is approved by the European Medicines Agency (EMA) and the FDA for the treatment of patients with GT. The approval of this drug has changed the landscape of treatment of GT and has allowed for better hemostatic outcomes in all patients, especially those who do not respond to platelet transfusions. Optimal dosing and interval of doses vary by center and clinical situation. Typical doses for acute bleeding are $90 \mathrm{mcg} / \mathrm{kg}$ intravenous (IV) every 2-6 hours until hemostasis is achieved (at least 3 doses). Perioperative dosing is $90 \mathrm{mcg} / \mathrm{kg}$ immediately before surgery and every two hours during the procedure, with doses every 2-6 hours postoperatively to prevent postsurgical bleeding. ${ }^{30}$ Effectiveness of this drug in the treatment of acute bleeding is high ${ }^{31}$ when started early in the course of bleeding and used in combination with other hemostatic treatment. It is also useful for perioperative management..$^{32}$ Although cases of thromboembolism during rFVIIa therapy in GT patients have occurred, it remains a safe medication in this population, with low rates of adverse reactions.

\section{Transfusion}

Patients with GT have a high risk of development of isoantibodies, with up to $30 \%$ of patients developing antiGPIIb/IIIa or anti-HLA antibodies after platelet transfusion..$^{33}$ Patients with pathogenic variants causing premature stop codons and leading to absent GPIIb/IIIa are at the highest risk of anti GPIIb/IIIa alloimmunization compared to those with other types of variants (81\% vs. $25 \%) .{ }^{34}$ Once these antibodies develop, the patient may no longer respond to platelet transfusion. For this reason, platelet transfusions should be reserved for only major surgeries, life-threatening bleeding, and significant bleeding that does not respond to the above measure. Transfusions in women of reproductive age should ideally be avoided as the antibodies can cross the placenta and affect the fetus. ${ }^{35}$

\section{Bone marrow transplant}

Allogeneic stem cell transplantation has been successfully performed in selected patients with severe recurrent bleeding using reduced intensity conditioning with good clinical outcomes. ${ }^{36,37}$ The presence in the recipient of antiplatelet antibodies affecting the graft remains a challenge in this patient population. ${ }^{38}$

\section{Pregnancy}

Pregnant women with GT have a high rate of complications and are best managed in a specialized center with a multidisciplinary team. While most complications relate to bleeding and occur at the time of delivery, management of the pregnant GT patients should start in the prenatal period. Counseling of the pregnancy-associated risks and screening of the father in consanguineous families to identify at-risk fetuses is important. Identification of HLA or GPIIb/IIIa antibodies during pregnancy, which are present in up to $70 \%$ of patients, is key for planning delivery. In general, regional anesthesia is contraindicated and support with rFVIIa and antifibrinolytics is given for vaginal deliveries with the option of adding platelet transfusion for cesarian sections. ${ }^{39}$ Primary post-partum hemorrhage is common, and a large proportion of women will require red cell transfusion. Clinicians should be aware that, given the phenotypic variability of this disorder, around half the women with GT who are pregnant may not be aware of the diagnosis. ${ }^{35}$

\section{Future directions}

Gene therapy is highly promising in providing a cure for patients with GT, with significant progress made using different techniques, vectors and model organisms. ${ }^{40-42}$ However, further advances that allow safe transgene delivery and stable expression in human models are still required. ${ }^{33}$

\section{Ongoing challenges}

Despite advances in the understanding of the pathophysiology, the relative ease of access to clinical laboratory techniques, and recent improvements in treatment options (such as rFVIIa), many challenges remain in the care of patients with this rare disorder. Access to clinicians and laboratories with the required expertise and resources to diagnose and treat GT is difficult, especially in areas of the world with limited resources. Large-scale data analysis and clinical research are problematic given the limited numbers of patients available and the highly constrained funding for rare disorders. Initiatives through the World Federation of Hemophilia for clinical care and multinational collaborations including the Glanzmann Thrombasthenia Registry for analysis of clinical outcomes, and sharing of molecular data through creation of publicly accessible databases have taken important steps in bridging the gaps. However, more investment is needed to guarantee timely access to quality care, until a true cure for the disease is developed. 


\section{References}

1. Commentary on and reprint of Glanzmann E, Hereditäre häemorrhagische thrombasthenie. Ein Beitrag zur Pathologie der Blutplättchen [Hereditary hemorrhagic thrombasthenia: A contribution on the pathology of blood platelets], in Jahrbuch für Kinderheilkunde (1918) 88:113-141. In: Lichtman MA, Spivak JL, Boxer LA, Shattil SJ, Henderson ES, eds. Hematology. San Diego: Academic Press, 2000:55-III.

2. Caen J, Cousin C. ["In vivo" disorder of platelet adhesiveness in Willebrand's disease and Glanzmann's thrombasthenias. Trial interpretation]. Nouv Rev Fr Hematol. 1962;2:685-694.

3. Caen JP, Castaldi PA, Leclerc JC, et al Congenital bleeding disorders with long bleeding time and normal platelet count: I. Glanzmann's thrombasthenia (report of fifteen patients). Am J Med. 1966;41(1):4-26.

4. Nurden AT, Caen JP. An abnormal platelet glycoprotein pattern in three cases of Glanzmann's thrombasthenia. $\mathrm{Br}$ J Haematol. 1974;28(2):253-260.

5. Phillips DR, Agin PP. Platelet membrane defects in Glanzmann's thrombasthenia. Evidence for decreased amounts of two major glycoproteins. J Clin Invest. 1977;60(3):535-545.

6. Disorders NOfR. NORD Resource Guide Orphan Disease Update [Newsletter] 2019 April 8, 2019 [cited 2019 April 22]; Available from: https://rarediseases.org

7. Nurden AT, Fiore M, Nurden P, Pillois X. Glanzmann thrombasthenia: a review of ITGA2B and ITGB3 defects with emphasis on variants, phenotypic variability, and mouse models. Blood. 2011;118(23):59966005.

8. Toogeh G, Sharifian R, Lak M, Safaee R, Artoni A, Peyvandi F. Presentation and pattern of symptoms in 382 patients with Glanzmann thrombasthenia in Iran. Am J Hematol. 2004;77(2):198-199.

9. George JN, Caen JP, Nurden AT. Glanzmann's thrombasthenia: the spectrum of clinical disease. Blood. 1990;75(7):13831395.

10. Marder VJ. Hemostasis and Thrombosis: Basic Principles and Clinical Practice: Wolters Kluwer Health, 2012.

11. Coller BS, Shattil SJ. The GPIIb/IIIa (integrin alphallbbeta3) odyssey: a technology-driven saga of a receptor with twists, turns, and even a bend. Blood. 2008:112(8):3011-3025

12. Coller BS, Seligsohn U, Peretz H, Newman PJ. Glanzmann thrombasthenia: new insights from an historical perspective. Semin Hematol. 1994;31(4):301-311.

13. Nurden AT, Pillois X. ITGA2B and ITGB3 gene mutations associated with Glanzmann thrombasthenia. Platelets. 2018;29(1):98101.

14. Richards S, Aziz N, Bale S, et al. Standards and guidelines for the interpretation of sequence variants: a joint consensus recommendation of the American College of Medical Genetics and Genomics and the Association for Molecular Pathology. Genet Med. 2015;17(5):405-424.

15. Kashiwagi H, Kunishima S, Kiyomizu K, et al. Demonstration of novel gain-of-function mutations of alphallbbeta3: association with macrothrombocytopenia and glanzmann thrombasthenia-like phenotype. Mol Genet Genomic Med. 2013;1(2):77-86.

16. Bury L, Malara A, Gresele P, Balduini A. Outside-in signalling generated by a constitutively activated integrin alphallbbeta3 impairs proplatelet formation in human megakaryocytes. PLoS One. 2012;7(4): e34449.

17. Nurden AT, Nurden P. Congenital platelet disorders and understanding of platelet function. Br J Haematol. 2014;165(2):165-178.

18. Lowe GC, Lordkipanidze M, Watson SP, UK GAPP study group. Utility of the ISTH bleeding assessment tool in predicting platelet defects in participants with suspected inherited platelet function disorders. J Thromb Haemost. 2013;11(9):1663-1668.

19. Harrison P. The role of PFA-100 testing in the investigation and management of haemostatic defects in children and adults. $\mathrm{Br} \mathrm{J}$ Haematol. 2005;130(1):3-10.

20. Gresele P, Subcommittee on Platelet Physiology of the International Society on T, Hemostasis. Diagnosis of inherited platelet function disorders: guidance from the SSC of the ISTH. J Thromb Haemost. 2015;13(2):314-322.

21. Al Ghaithi R, Drake S, Watson SP, Morgan NV, Harrison P. Comparison of multiple electrode aggregometry with lumi-aggregometry for the diagnosis of patients with mild bleeding disorders. J Thromb Haemost. 2017;15(10):2045-2052

22. Miller JL. Glycoprotein analysis for the diagnostic evaluation of platelet disorders Semin Thromb Hemost. 2009;35(2):224 232.

23. Kuijpers TW, van de Vijver E, Weterman $M A$, et al. LAD-1/variant syndrome is caused by mutations in FERMT3. Blood. 2009;113(19):4740-4746.

24. Westbury SK, Canault M, Greene D, et al. Expanded repertoire of RASGRP2 variants responsible for platelet dysfunction and severe bleeding. Blood. 2017;130(8):10261030

25. Tholouli E, Hay CR, O'Gorman P, Makris M. Acquired Glanzmann's thrombasthenia without thrombocytopenia: a severe acquired autoimmune bleeding disorder. $\mathrm{Br} \mathrm{J}$ Haematol. 2004;127(2):209-213.

26. Nurden AT. Acquired Glanzmann thrombasthenia: From antibodies to anti-platelet drugs. Blood Rev. 2019;36:10-22.

27. Nurden AT. Glanzmann thrombasthenia. Orphanet J Rare Dis. 2006;1:10.

28. Resource CG. [cited 2019 May 2]; Available from: https://www.clinicalgenome.org/

29. Grainger JD, Thachil J, Will AM. How we treat the platelet glycoprotein defects; Glanzmann thrombasthenia and Bernard Soulier syndrome in children and adults. $\mathrm{Br}$ Haematol. 2018;182(5):621-632.

30. Inc NN. NovoSeven RT [package insert]. Plainsboro, NJ; 2019.

31. Poon MC, D'Oiron R, Von Depka M, et al Prophylactic and therapeutic recombinant factor VIIa administration to patients with Glanzmann's thrombasthenia: results of an international survey. J Thromb Haemost. 2004;2(7):1096-1103.

32. Poon MC, d'Oiron R, Zotz RB, et al. The international, prospective Glanzmann Thrombasthenia Registry: treatment and outcomes in surgical intervention. Haematologica. 2015;100(8):1038-1044.

33. Poon MC, Di Minno G, d'Oiron R, Zotz R New Insights Into the Treatment of Glanzmann Thrombasthenia. Transfus Med Rev. 2016;30(2):92-99.

34. Fiore M, Firah N, Pillois X, Nurden P, Heilig R, Nurden AT. Natural history of platelet antibody formation against alphallbbeta3 in a French cohort of Glanzmann thrombasthenia patients. Haemophilia. 2012;18(3): e201-209.

35. Siddiq S, Clark A, Mumford A. A systematic review of the management and outcomes of pregnancy in Glanzmann thrombasthenia. Haemophilia. 2011;17(5):e858-869.

36. Bellucci S, Damaj G, Boval B, et al. Bone marrow transplantation in severe Glanzmann's thrombasthenia with antiplatelet alloimmunization. Bone Marrow Transplant. 2000;25(3):327-330

37. Ramzi M, Dehghani M, Haghighat S, Nejad HH. Stem Cell Transplant in Severe Glanzmann Thrombasthenia in an Adult Patient. Exp Clin Transplant. 2016;14(6):688690.

38. Nurden AT, Pillois X, Wilcox DA Glanzmann thrombasthenia: state of the art and future directions. Semin Thromb Hemost. 2013;39(6):642-655.

39. Bolton-Maggs PH, Chalmers EA, Collins PW, et al. A review of inherited platelet disorders with guidelines for their managemen on behalf of the UKHCDO. Br J Haematol. 2006;135(5):603-633.

40. Wilcox DA, Olsen JC, Ishizawa L, et al Megakaryocyte-targeted synthesis of the integrin beta(3)-subunit results in the phenotypic correction of Glanzmann thrombasthenia. Blood. 2000;95(12):3645-3651

41. Fang J, Hodivala-Dilke K, Johnson BD, et al Therapeutic expression of the platelet-specific integrin, alphallbbeta3, in a murine model for Glanzmann thrombasthenia. Blood. 2005;106(8):2671-2679.

42. Sullivan SK, Mills JA, Koukouritaki SB, et al. High-level transgene expression in induced pluripotent stem cell-derived megakaryocytes: correction of Glanzmann thrombasthenia. Blood. 2014;123(5):753-757. 